\section{Share and share alike}

Humans harbor a vast and diverse population of microbes that are affected by many factors, including age and genetics, and can in turn influence immunological status. In eLife, Knight and colleagues undertake a comprehensive survey of microbes that inhabit the skin, mouth and gut to determine the influence of cohabitation with family members and dogs. Many patterns emerge; for example, members of the same household share more microbiota with each other than with people from other households, and this overlap is greatest for the skin microbiota. Notably, dog ownership results in more sharing of microbiota among humans, and there is also considerable sharing of microbiota between dogs and humans. Age also has a role, with the greatest changes in gut microbiota occurring within a window during early infancy but the skin microbiota remaining relatively stable with age. A shared environment therefore strongly influences microbiota colonization and this might potentially feed forward into 'tuning' of the immune system. eLife 2, e00458 (2013)

\section{Restricting T cell responses}

ILCs have effector cytokine profiles similar to those of $\mathrm{CD}^{+} \mathrm{T}$ cells but are much less numerous than are $\mathrm{CD} 4^{+} \mathrm{T}$ cells. In Nature, Hepworth et al. specifically target ROR $\gamma \mathrm{t}^{+}$ILCs (group 3 ILCs) by genetic or antibody-mediated approaches to assess the specific role of this lymphoid population in the induction of immune responses and adaptive immunity. The authors demonstrate a critical role for ILCs in the regulation of inflammatory adaptive immune responses to commensal bacteria that is independent of the effector cytokines IL-22, IL-17A and IL-23. In contrast to ILCs of groups 1 and 2, mouse and human ROR $\gamma \mathrm{t}^{+}$ILCs express MHC class II and can process and present antigen. However, they lack expression of the costimulatory molecules CD40, CD80 and CD86 and inhibit the proliferation of $\mathrm{CD}^{+} \mathrm{T}$ cells through MHC class IIdependent interactions. Deletion of MHC class II on ROR $\gamma \mathrm{t}^{+}$ILCs leads to commensal bacteria-driven pathological $\mathrm{CD} 4^{+} \mathrm{T}$ cell responses and intestinal inflammation.

Nature (22 May 2013) doi:10.1038/nature12240

\section{Commensal help}

Toxoplasma gondii causes acute mucosal infections that are controlled by Ly6C+ inflammatory monocytes. In Nature Medicine, Grainger et al. show that $\mathrm{Ly} 6 \mathrm{C}^{+}$monocytes serve additional roles by suppressing collateral tissue damage that results from unstrained neutrophil activation. $C c r 2^{-1-}$ mice, which have defects in circulating Ly $6 \mathrm{C}^{+}$monocytes, have more severe gut pathology after infection with $T$. gondii. Commensal bacteria that escape into gut tissues elicit production of prostaglandin $\mathrm{E}_{2}\left(\mathrm{PGE}_{2}\right)$ by Ly $6 \mathrm{C}^{+}$monocytes. $\mathrm{PGE}_{2}$ inhibits the release of TNF and reactive oxygen from neutrophils in infected tissues. Inhibition of $\mathrm{PGE}_{2}$ production similarly exacerbates tissue pathology but does not alter monocyte control of $T$. gondii. Thus, exposure to gut bacteria induces protective properties of $\mathrm{Ly}_{6 \mathrm{C}} \mathrm{C}^{+}$monocytes to limit tissue damage during acute infection.

Nat. Med. (26 May 2013) doi:10.1038/nm.3189

Written by Laurie A. Dempsey, Zoltan Fehervari \& Ioana Visan

\section{Seeking advantages}

Salmonella serovar Typhimurium causes acute cecal inflammation and takes advantage of host immune responses to establish infection. In PLoS Pathogens, Bäumler and colleagues find that host inflammation confers enhanced competitive fitness to bacteria that express Tsr and Aer, two methyl-accepting chemotaxis proteins. The generation of reactive oxygen species by neutrophils leads to the production of tetrathionate, a sulfur compound that Salmonella can use as an electron acceptor during anaerobic respiration. Nitric oxide is also produced during inflammation and reacts with reactive oxygen species to generate nitrates. Aer and Tsr are able to sense higher concentrations of tetrathionate and nitrate, respectively, and direct chemotaxis toward their source at the mucosal epithelium. Because of their ability to use such molecules as electron acceptors in respiration, Salmonella have a growth advantage over resident bacteria, conferred by Aer and Tsr. Thus, unwittingly, host inflammatory responses aid infecting Salmonella by creating unique niches that the bacteria exploit.

PLoS Pathog. 9. e1003267 (18 April 2013)

$L A D$

\section{Mucosal ILC diversity}

The gastrointestinal mucosa harbors diverse innate lymphoid cell (ILC) subsets that secrete specific cytokines early during the immune response. In Immunity, Fuchs et al. identify a subset of intraepithelial ILCs that are $\mathrm{NKp} 44^{+} \mathrm{CD} 103^{+}$in humans and $\mathrm{NKp} 46^{+} \mathrm{NK} 1.1^{+} \mathrm{CD} 160^{+}$in mice and are distinct from classical NK cells, although they belong to the same group of ILC1 cells. These cells produce IFN- $\gamma$ when stimulated with IL-12 and IL-15, can release lytic mediators, have an intraepithelial location and express markers consistent with TGF- $\beta$ exposure and activation. In mice, they develop independently of IL-15 signaling, which confirms that they are not conventional NK cells. These cells are responsive to danger signals from epithelial and myeloid cells and proliferate in the small intestine epithelium in patients with Crohn's disease, which suggests that they may have a role in intestinal inflammation and immunopathology.

\title{
IL-22 controls transmissible colitis
}

IL-22 is an important cytokine at barrier surfaces that triggers the production of antimicrobial peptides and mucus. Flavell and colleagues in the Journal of Immunology use an IL-22-deficient mouse to demonstrate that this cytokine shapes the population of colonic microbes to prevent colitogenicity. IL-22-deficient mice have ostensibly normal colonic morphology and do not manifest spontaneous inflammation; however, induced colitis is worse in those mice than in wild-type mice. Furthermore, wild-type mice cohoused with IL-22-deficient mice have more severe induced colitis, which suggests that the colitis is transmissible. Pyrosequencing indicates that the microbial phyla in IL-22-deficient mice differs from that of wild-type mice and, more notably, the microbiota of wild-type mice cohoused with IL-22-deficient mice more closely resembles that of the IL-22-deficient mice. As expected, IL-22-deficient mice have a lower abundance of the antimicrobial peptides RegIII $\beta$ and RegIII $\gamma$ but, curiously, so do wild-type mice cohoused with those mice. This suggests that the microbiota is both shaped by IL-22 and, in turn, also influences its expression.

J. Immunol. (12 April 2013) doi:10.4049/jimmunol.1300016 